\title{
Envelhecimento e isolamento social: uma revisão integrativa
}

\author{
Aging and social isolation: an integrative review \\ Envejecimiento y aislamiento social: una revisión integradora \\ Patricia Araújo Bezerra ${ }^{1}$ io https://oridi.org//0000-0003-2696-576x \\ José Walter Nunes ${ }^{2}$ io https://orcid.org/0000-0003-0827-3882 \\ Leides Barroso de Azevedo Moura² io https://orcid.org/0000-0002-1208-4569
}

Como citar:

Bezerra PA, Nunes JW, Moura LB.

Envelhecimento e isolamento social: uma revisão integrativa. Acta Paul Enferm. 2021;34:eAPE02661.

DOI

http://dx.doi.org/10.37689/actaape/2021AR02661

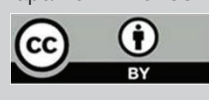

Descritores

Envelhecimento; Idoso; Ageísmo; Isolamento social; Solidão; Comportamnto social

Keywords

Aging; Aged; Ageism; Social isolation; Loneliness;

Social behavio

Descriptores

Envejecimiento; Anciano; Ageísmo; Aislamiento social; Soledad; Conducta social

Submetido

12 de Setembro de 2019

Aceito

1 de Junho de 2020

Autor correspondente

Patricia Araújo Bezerra

E-mail: pat.araujobezerra@gmail.com

\section{Resumo}

Objetivo: Analisar os conceitos disponíveis na literatura sobre o isolamento social de pessoas idosas.

Métodos: Revisão de literatura do tipo integrativa baseada na perspectiva teórica do ageísmo. Os dados foram coletados nos meses de março a maio de 2019, nas bases LILACS, MEDLINE, EBSCO e Web of Science. Utilizou-se o cruzamento das palavras-chave "Older adult" e "Elderly", com os descritores controlados "Aged", "Social Isolation" e "Loneliness", registrados no Medical Subject Headings (MeSH), com o uso do operador booleano AND. A amostra final foi composta por 18 artigos.

Resultados: 0 isolamento social da pessoa idosa relaciona-se com a ausência ou inadequação da família, amigos e redes sociais gerais; isolamento não significa necessariamente solidão; a operacionalização do conceito de isolamento social necessita de padronização para uma melhor mensuração; há fatores de risco já identificados e possíveis consequências; há intervenções, mas insuficiente informação se elas se baseiam em evidências; descreve-se a relação entre isolamento e solidão e apresentam-se fatores protetivos, porém com baixo nível de evidência.

Conclusão: Conclui-se que há necessidade de criação de instrumentos para operacionalizar o conceito de isolamento social, uma vez que os estudos apresentam uma heterogeneidade de conceitos e embasamentos teóricos.

\section{Abstract}

Objective: To analyze the concepts available in the literature about the social isolation of older people.

Methods: This was an integrative literature review with basis on the theoretical perspective of ageism. The data were collected from March to May 2019, in LILACS, MEDLINE, EBSCO and Web of Science databases. The crossing of keywords "Older adult" and "Elderly" were used, with the controlled descriptors "Aged," "Social Isolation," and "Loneliness" registered in the Medical Subject Headings (MeSH), using the Boolean operator AND. The final sample consisted of 18 articles.

Results: The social isolation of older person is related to absence or inadequacy of family, friends and general social networks; isolation does not necessarily mean loneliness; operationalization of the social isolation concept needs standardization for a better measurement; risk factors was already identified with possible consequences; interventions are available, but there is insufficient information whether they are based on evidence; the relationship between isolation and loneliness is described and protective factors are presented, but with a low level of evidence.

Conclusion: Instruments to operationalize the concept of social isolation need to be created, as the studies present heterogeneity of concepts and theoretical bases. 


\section{Resumen}

Objetivo: Analizar los conceptos disponibles en la literatura sobre el aislamiento social de personas mayores.

Métodos: Revisión integradora de literatura basada en la perspectiva teórica del edadismo. Los datos fueron recopilados entre los meses de marzo y mayo de 2019, en las bases LILACS, MEDLINE, EBSCO y Web of Science. Se utilizó el cruce de las palaras clave "Older adult" y "Elderly", con los descriptores controlados "Aged", "Social Isolation" y "Loneliness", registrados en el Medical Subject Headings (MeSH), con el operador booleano AND. La muestra final estuvo compuesta por 18 artículos.

Resultados: El aislamiento social de la persona mayor se relaciona con la ausencia o falta de capacidad de la familia, amigos y redes sociales generales. Aislamiento no significa soledad necesariamente. La funcionalidad del concepto de aislamiento social necesita estandarización para obtener una mejor medición. Existen factores de riesgo ya identificados y sus posibles consecuencias. Hay intervenciones, pero sin suficiente información si se basan en evidencias. Se describe la relación entre aislamiento y soledad y se presentan factores protectores, pero con bajo nivel de evidencia.

Conclusión: Se concluye que hay una necesidad de crear instrumentos para operacionalizar el concepto de aislamiento social, dado que los estudios presentan una heterogeneidad de conceptos y fundamentos teóricos.

\section{Introdução}

O processo de envelhecimento populacional já é uma realidade no Brasil. Pela primeira vez, a maioria das pessoas pode esperar viver até os 60 anos ou mais. ${ }^{(1)}$ No entanto, viver mais não significa viver melhor. A dinâmica das cidades e de trabalho, os novos arranjos de moradia e família, o crescente culto ao individualismo e mudanças nos valores tradicionais da sociedade influenciam de modo desigual e contraditório na qualidade de vida das pessoas. ${ }^{(2,3)}$

Entre os fatores que têm se destacado como um potencial problema de saúde pública encontra-se o isolamento social. ${ }^{(4)}$ Este pode ser definido como um estado no qual indivíduos experimentam cada vez menos envolvimento social do que gostariam com outras pessoas. Isso interfere na sua qualidade de vida. Refere-se à objetiva separação de outros indivíduos, ocasionando ausência ou poucas interaçôes sociais no dia a dia. ${ }^{(5)}$ Este fenômeno, embora possa ser experimentado em qualquer fase da vida, é mais prevalente entre pessoas idosas, com estimativa em torno de $10 \%$ a $43 \%$, a depender do percurso histórico e das condiçóes de vida. ${ }^{(6)}$ A vivência do isolamento pode ocorrer conjuntamente com a sensação subjetiva de solidão ou mesmo pode gerá-la como consequência. ${ }^{(7)}$ Esta realidade tem demonstrado ser um problema multifacetado. Há estudo que afirma que ela está associada ao aumento da mortalidade por todas as causas. ${ }^{(8)}$

No Brasil, cujas territorialidades são marcadas por expressivas variabilidades nas condiçóes de vida de pessoas idosas, como as características sociais, econômicas, demográficas, culturais e ambientais heterogêneas, os impactos na interação social podem ser bastante diferentes e, consequentemente, a longevidade e a qualidade de vida dos adultos idosos. O entendimento dessa problemática contribui para ampliar a promoção de políticas que busquem espaços de sociabilidade e de interação entre idosos, famílias e cuidadores e, mais que isso, a fim de ressignificar a formação dos profissionais de saúde para o cuidado.

A magnitude do envelhecimento populacional é crescente e influenciada por diferentes fatores, e está relacionada com desiguais e contraditórias formas de viver a idade avançada, tendo a interação social como um marcador para a qualidade de vida. Este estudo teve como objetivo analisar os conceitos disponíveis na literatura sobre o isolamento social de pessoas idosas

\section{Métodos}

Realizou-se uma revisão integrativa de literatura composta por seis etapas. ${ }^{(9)}$ No primeiro momento, adotou-se a pergunta de pesquisa: Quais conceitos e perspectivas estão disponíveis na literatura acerca do isolamento social de pessoas idosas? $\mathrm{Na}$ segunda etapa, estabeleceram-se os critérios de inclusão: (i) apresentar fatores que contribuem para o isolamento social de idosos; (ii) abordar aspectos relacionados aos fatores de risco à saúde; (iii) apresentar perspectivas de abordagem sobre o isolamento social de pessoas idosas; iv) artigos nas línguas inglesa, espanhola e portuguesa. Os critérios de exclusão foram: (i) artigos de revisão; (ii) textos, cartas, te- 
ses, dissertaçóes e artigos não indexados em revistas científicas; (iii) não apresentar no artigo o conceito ou definiçóes de isolamento social; (iv) abordar exclusivamente a solidão.

A busca dos artigos foi realizada nas bases de dados Web ofScience, EBSCO, LILACS e MEDLINE, estas duas últimas por intermédio de consulta na Latin American and Caribbean Center on Health Sciences Information. A busca no EBSCO incluiu a base de dados CINAHL e, os achados foram apresentados como quantitativo total da EBSCO, no período de março a maio de 2019. Foram consultados por intermédio do cruzamento das palavras-chave "Older adult" e "Elderly", com os descritores controlados "Aged", "Social Isolation" e "Loneliness", registrados no Medical Subject Headings (MeSH), utilizando-se MESH + All fields, com o uso do operador booleano AND. Utilizou-se o termo "elderly" (idoso), considerado preconceituoso segundo a perspectiva teórica que embasa o ageísmo, porque o DECS e o MESH ainda apresentam vários artigos que utilizam esse descritor quando tratam do envelhecimento. Não houve limite de ano de publicação. $\mathrm{O}$ estudo seguiu as recomendaçóes do Preferred Reporting Items for Systematic reviews and Meta-Analyses (PRISMA).
Identificou-se um total de 216 dados de seleção, sendo 82 da Web of Science, 48 da EBSCO, 2 da Lilacs e 84 da Medline. A primeira etapa do processo de seleção de literatura incluiu a leitura dos títulos e os resumos dos estudos, sendo excluídos 148 títulos por não cumprirem o objeto da pergunta orientadora e os critérios de inclusão descritos. Um total de 23 artigos achava-se em duplicidade e foram removidos. Posteriormente, procedeu-se à leitura completa de 45 artigos selecionados. Após aplicação dos critérios de exclusão, obteve-se como amostra final um total de 18 artigos (Figura 1). No decorrer das etapas, as eventuais discordâncias na seleção de artigos foram resolvidas por dois juízes com conhecimento no tema da pesquisa, que validaram a escolha dos artigos selecionados na amostra final.

A seguir, procedeu-se a uma nova leitura na íntegra dos artigos da amostra, a partir de formulário pré-estruturado, em que constam: autores, ano, local, delineamento do estudo, conceito de isolamento social, escalas utilizadas e principais resultados referentes ao isolamento social. Após a sistematização, procedeu-se à análise qualitativa ${ }^{(10)}$ com leitura dos achados e extração dos fragmentos, conforme apresentados nos resultados e nas consideraçóes finais. Utilizou-se para apoio à análise o software Iramutec 0.7 alpha 2. Por fim,

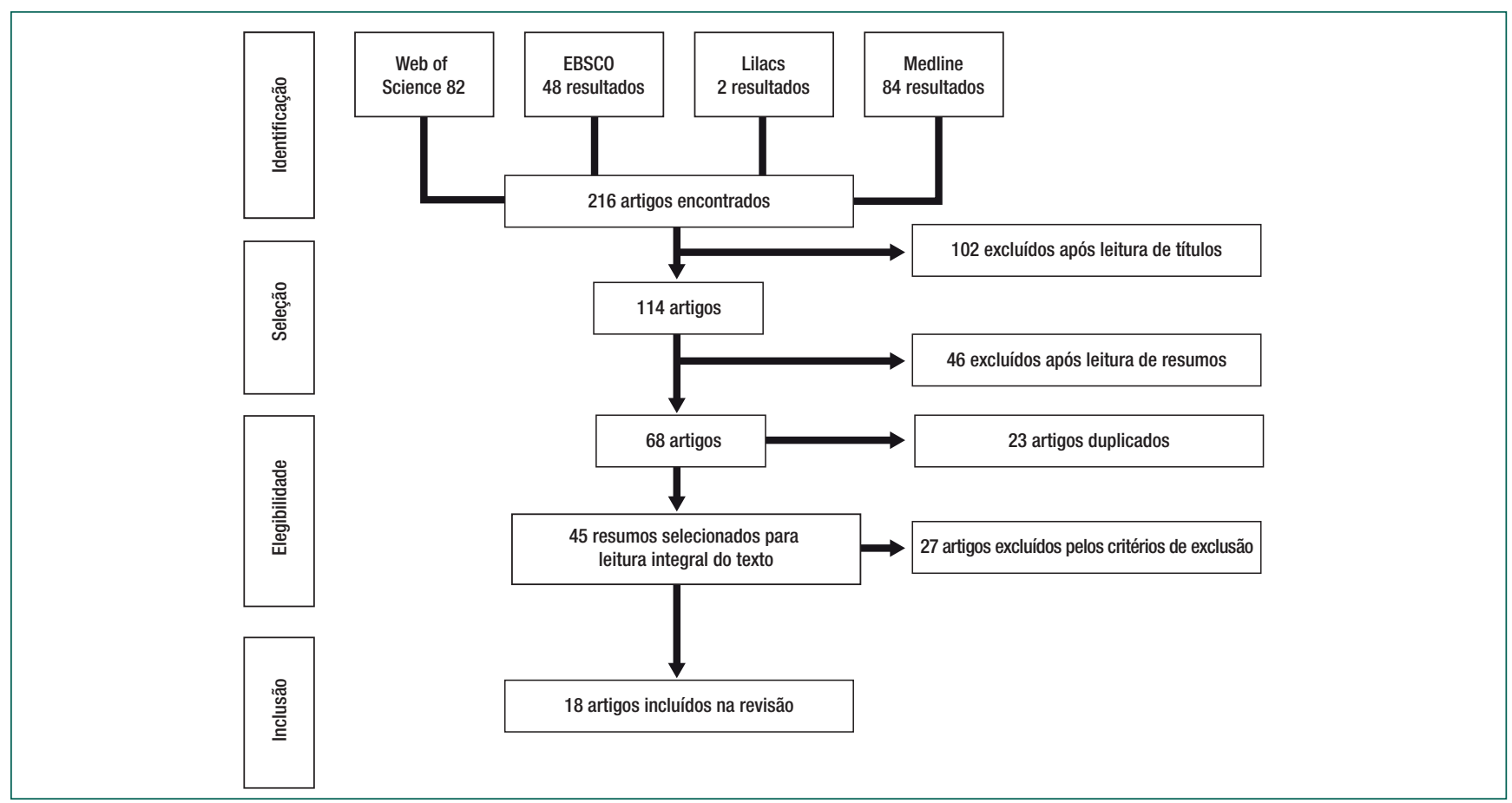

Figura 1. Base de dados e estratégia de busca selecionada 
consideraram-se os aspectos éticos, a autoria das ideias, os conceitos e as definiçôes presentes nos artigos.

\section{Resultados}

Foram selecionados 18 artigos na amostra final; todos estavam escritos na língua inglesa ( $28 \%$ originários dos EUA, 28\% da Inglaterra e os demais, 5,5\% cada: Índia, Holanda, Itália, Japão, Malásia, Países Baixos e Taiwan). $\mathrm{O}$ ano de publicação variou de 2009 a 2019. O ano com maior número de publicaçóes foi 2013, com 22\%.

Os estudos analisados indicam como fatores associados ao risco de isolamento social de pessoas idosas tanto questóes individuais quanto sociais, podendo ser assim elencados:

1. Fatores relacionados à saúde física e mental do indivíduo: pessoas com dependência física ou comprometimento funcional, surdez ou perda auditiva, doenças neurológicas e doenças de longa duração limitadas; ${ }^{(11-21)}$
2. Fatores sociodemográficos e baseados em iniquidades sociais: ser mulher, solteiro ou viúvo, vivenciou perda de pessoas ou entes próximos, desempregado ou economicamente dependente, com baixa escolaridade, baixa competência em saúde, baixo poder de decisão, baixa renda familiar, sem filhos, menor tamanho de arranjo familiar, com pouca ou ausente vinculação religiosa, morar em área rural e questôes geográficas, baixa mobilidade e acesso a transportes, sem acesso a um sistema de previdência social, morar em locais de cuidado residenciais ou instituições de longa permanência, estar hospitalizado; $(15,17,18,20,21-27)$

3. Fatores contextuais e estruturais: como políticas econômicas e sociais que produzem e mantêm desigualdades socioeconômicas que limitam a oportunidade de participação em atividades sociais. ${ }^{(18)}$

Todos os artigos apresentaram alguma definição conceitual para isolamento social, conforme apresentado no quadro 1.

Quadro 1. Síntese das definições conceituais sobre isolamento social e principais conclusões segundo ano, país de publicação e delineamento do estudo

\begin{tabular}{|c|c|c|c|}
\hline Ano e local & Delineamento do estudo & Conceito de isolamento social & Principais conclusões \\
\hline $\begin{array}{l}\text { (2018) } \\
\text { Taiwan }^{(11)}\end{array}$ & $\begin{array}{l}\text { Estudo prospectivo baseado em pesquisa } \\
\text { Iongitudinal "Meio Social e Biomarcadores do } \\
\text { Envelhecimento em Taiwan" }\end{array}$ & $\begin{array}{l}\text { Aspecto objetivo das relações sociais, } \\
\text { tamanho da rede, diversidade e frequência } \\
\text { de contatos. }\end{array}$ & $\begin{array}{l}0 \text { isolamento social está associado à pior qualidade do sono em adultos mais } \\
\text { velhos e indica que esse efeito é independente do sentimento subjetivo de solidão. }\end{array}$ \\
\hline $\begin{array}{l}(2009) \\
E^{\prime} A^{(12)}\end{array}$ & $\begin{array}{l}\text { Estudo sobre propriedades psicométricas de } \\
\text { escalas de Desconexão Social e Isolamento } \\
\text { Social, baseado em dados da pesquisa } \\
\text { "Projeto Nacional de Vida Social, Saúde e } \\
\text { Envelhecimento" (NSHAP). }\end{array}$ & $\begin{array}{l}\text { Desconexão social (separação física dos } \\
\text { outros) e isolamento social percebido } \\
\text { (sentimentos de solidão e falta de apoio } \\
\text { social). } \\
\text { Indivíduos sem conexão social tendem a se } \\
\text { sentir isolados. }\end{array}$ & $\begin{array}{l}\text { Indivíduos que possuem pequenas redes sociais ou raramente participam de } \\
\text { atividades sociais não necessariamente se sentem solitários. A pessoa pode estar } \\
\text { cercada de amigos e familiares, mas percebe a falta de apoio social e se sente } \\
\text { excluída. Inconclusivo sobre se } 0 \text { isolamento social aumenta com a idade. }\end{array}$ \\
\hline $\begin{array}{l}(2013) \\
E^{\prime} A^{(13)}\end{array}$ & $\begin{array}{l}\text { Estudo transversal desenvolvido em } \\
\text { instituições para pessoas idosas no Alabama. }\end{array}$ & $\begin{array}{l}\text { Experiência objetiva de ausência de contato } \\
\text { com outras pessoas, especialmente de } \\
\text { pessoas que oferecem o suporte social } \\
\text { necessário ou desejado. Isolamento social } \\
\text { é a ausência de relacionamento social } \\
\text { significativo. }\end{array}$ & $\begin{array}{l}\text { A frequência de uso da internet afeta a solidão, mas não as percepções de } \\
\text { isolamento, indicando uma maior frequência associada a níveis mais baixos de } \\
\text { solidão, mas não com níveis mais baixos de isolamento social. }\end{array}$ \\
\hline $\begin{array}{l}(2012) \\
E^{\prime} A^{(14)}\end{array}$ & $\begin{array}{l}\text { Estudo de indicadores de isolamento social } \\
\text { baseado nos dados da Pesquisa Longitudinal } \\
\text { "Estudo em Saúde e Aposentadoria", } \\
\text { desenvolvido pela Universidade de Michigan. }\end{array}$ & $\begin{array}{l}\text { Falta objetiva de relacionamentos e interação } \\
\text { social. }\end{array}$ & $\begin{array}{l}0 \text { isolamento e a solidão não são altamente correlacionados entre si, o que implica } \\
\text { que os dois construtos são distintos. Os impactos na saúde física e mental podem } \\
\text { ter diferentes repercussões. Para intervenção é necessário distingui-los. }\end{array}$ \\
\hline $\begin{array}{l}(2017) \\
E^{\prime} A^{(15)}\end{array}$ & $\begin{array}{l}\text { Estudo transversal desenvolvido com pessoas } \\
\text { idosas residentes em moradias subsidiadas } \\
\text { pelo governo. }\end{array}$ & $\begin{array}{l}0 \text { isolamento social está associado à falta de } \\
\text { recursos sociais (amigos, familiares e relações } \\
\text { de confiança), o que resulta em piores } \\
\text { resultados de saúde física e mental. }\end{array}$ & $\begin{array}{l}0 \text { escore médio para residentes idosos de habitações acessíveis à classe } \\
\text { econômica mais baixa revelou que quase metade dos moradores era socialmente } \\
\text { isolada ou possuía "alto risco". }\end{array}$ \\
\hline $\begin{array}{l}\text { (2012) } \\
\text { Itália(16) }^{(16)}\end{array}$ & $\begin{array}{l}\text { Estudo longitudinal com idosos hospitalizados, } \\
\text { utilizando o "Levantamento geriátrico global". }\end{array}$ & $\begin{array}{l}\text { Definido como ausência ou diminuição de } \\
\text { redes sociais mais próximas e/ou apoio social } \\
\text { informal. }\end{array}$ & $\begin{array}{l}\text { Para indivíduos hospitalizados, a qualidade de vida foi um preditor independente de } \\
\text { isolamento; a mulher teve duas vezes mais risco de hospitalização; o isolamento } \\
\text { significou maior chance de re-hospitalização. }\end{array}$ \\
\hline $\begin{array}{l}\text { (2013) } \\
\text { Malásia' }^{(17)}\end{array}$ & $\begin{array}{l}\text { Estudo baseado em dados da pesquisa } \\
\text { nacional "Padrões de Relacionamentos } \\
\text { Sociais e Bem-Estar Psicológico entre } \\
\text { pessoas idosas da Malásia". }\end{array}$ & $\begin{array}{l}\text { Isolamento social definido objetivamente como } \\
\text { ausência de contato ou interação com outras } \\
\text { pessoas, e subjetivamente como sentimento } \\
\text { de solidão ou ausência de companhia ou } \\
\text { comunicação genuína com outras pessoas. }\end{array}$ & $\begin{array}{l}\text { Número de filhos, irmãos, tamanho do agregado familiar, autoavaliação de saúde, } \\
\text { local de residência, propriedade, sexo e etnia foram significativamente associados } \\
\text { ao isolamento social. }\end{array}$ \\
\hline
\end{tabular}




\begin{tabular}{|c|c|c|c|}
\hline $\begin{array}{l}\text { (2018) } \\
\text { Índia } \\
\text { (18) }\end{array}$ & $\begin{array}{l}\text { Estudo baseado em dados da Pesquisa } \\
\text { "Construindo conhecimento sobre } \\
\text { envelhecimento populacional na Índia" } \\
\text { (BKPAl). }\end{array}$ & $\begin{array}{l}\text { Isolamento social ocorre quando, nos últimos } \\
12 \text { meses, a pessoa idosa não participou de } \\
\text { encontro público; de reunião de grupo/clube/ } \\
\text { organização; de programa religioso, nem } \\
\text { recebeu visita de amigo ou de familiares. }\end{array}$ & $\begin{array}{l}\text { Ausência de benefícios previdenciários, Alzheimer e possuir dependência de AVD } \\
\text { aumentam o risco para isolamento. Ser cristão ativo ou possuir de três a cinco } \\
\text { filhos diminui o risco. Associação com câncer não foi significativa. }\end{array}$ \\
\hline $\begin{array}{l}(2015) \\
\text { Países } \\
\text { Baixos }\end{array}$ & $\begin{array}{l}\text { Estudo com abordagem qualitativa mediante } \\
\text { entrevistas em profundidade. }\end{array}$ & $\begin{array}{l}\text { A definição de isolamento social parte do } \\
\text { contexto de políticas do Estado de bem-estar } \\
\text { social assumido pelos países europeus } \\
\text { ocidentais, que enfatiza a importância da } \\
\text { independência e da autoconfiança dos } \\
\text { cidadãos e apresenta uma tipologia de } \\
\text { base teórica de intervenções que ampliam } \\
\text { a autoconfiança de pessoas idosas } \\
\text { consideradas socialmente isoladas. }\end{array}$ & $\begin{array}{l}0 \text { isolamento social foi percebido a partir de duas classificações: após evento de } \\
\text { vida recente - situacional -, e os indivíduos que estão isolados há muito tempo } \\
\text { - estrutural. Motivaçōes de mudança e suporte são mais requeridas no caso } \\
\text { estrutural. }\end{array}$ \\
\hline $\begin{array}{l}\text { (2019) } \\
\text { Canadá(20) }\end{array}$ & $\begin{array}{l}\text { Estudo transversal baseado em dados } \\
\text { nacionais da Pesquisa Longitudinal } \\
\text { Canadense sobre Envelhecimento (CLSA) e } \\
\text { do Censo 2016, com estatística inferencial de } \\
\text { regressão logística multinível. }\end{array}$ & $\begin{array}{l}\text { Contato e laços que indivíduos possuem nas } \\
\text { redes sociais, que vão do mais próximo ao } \\
\text { menos próximo, baseado no "Modelo Comboio } \\
\text { de Relacionamento Social". }\end{array}$ & $\begin{array}{l}\text { Idade, sexo, escolaridade, renda, comprometimento funcional, doenças crônicas } \\
\text { foram relacionados tanto ao isolamento social quanto à solidão. Ter } 65 \text { anos ou } \\
\text { mais com baixa renda pode aumentar a relação com isolamento. }\end{array}$ \\
\hline $\begin{array}{l}\text { (2016) } \\
\text { Canadá(21) }\end{array}$ & $\begin{array}{l}\text { Estudo quantitativo de base populacional, } \\
\text { envolvendo pessoas de } 45 \text { anos ou mais, } \\
\text { chamada "Pesquisa Canadense Comunitária } \\
\text { de Saúde sobre Envelhecimento Saudável } \\
\text { (CCHS-HA)". }\end{array}$ & $\begin{array}{l}0 \text { isolamento social mesclava variável que } \\
\text { media solidão e senso de pertencimento } \\
\text { comunitário por duas escalas. } \\
0 \text { participante é considerado socialmente } \\
\text { isolado se ele se sente só e apresenta um } \\
\text { fraco senso de pertencimento comunitário. }\end{array}$ & $\begin{array}{l}0 \text { isolamento aumenta com deficiência auditiva entre as mulheres, e não } \\
\text { entre homens. Idade, escolaridade, convivência, regularidade motora, trabalho, } \\
\text { incontinência, medo de cair e limitaçôes funcionais aumentaram as chances. }\end{array}$ \\
\hline $\begin{array}{l}(2012) \\
\text { Japãa(122) }\end{array}$ & $\begin{array}{l}\text { Estudo Quasi-Experimental para testar um } \\
\text { programa de enfrentamento do isolamento } \\
\text { social para idosos japoneses com grupo de } \\
\text { intervenção e grupo controle, pré-teste antes } \\
\text { da intervenção, amostragem aleatória e coleta } \\
\text { de dados no primeiro e no sexto mês após a } \\
\text { intervenção. } \\
\end{array}$ & $\begin{array}{l}\text { Qualitativas e quantitativas imperfeições nas } \\
\text { interações sociais associadas à escassez } \\
\text { de relacionamentos. Correlaciona-se com } \\
\text { o conceito de suporte social emocional e } \\
\text { instrumental. }\end{array}$ & $\begin{array}{l}\text { Programas destinados a prevenir o isolamento social são eficazes quando } \\
\text { utilizam os recursos existentes na comunidade; são feitos levando em conta as } \\
\text { necessidades do indivíduo e têm como alvo pessoas que podem compartilhar } \\
\text { experiências semelhantes. }\end{array}$ \\
\hline $\begin{array}{l}(2019) \\
\text { Inglaterra }\end{array}$ & $\begin{array}{l}\text { Estudo quantitativo baseado em dados da } \\
\text { Pesquisa Longitudinal de Envelhecimento } \\
\text { Inglês (ELSA) com pessoas inglesas de } 50 \\
\text { anos ou mais, da sexta fase de coleta de } \\
\text { dados (2012-2013). }\end{array}$ & $\begin{array}{l}\text { Isolamento social assumido como ausência } \\
\text { de contato mensal regular com familiares e } \\
\text { amigos, bem como ausência de envolvimento } \\
\text { em atividades de organizações sociais. }\end{array}$ & $\begin{array}{l}0 \text { isolamento está relacionado à redução da atividade física objetiva diária e ao } \\
\text { maior tempo sedentário. Isso pode contribuir para o aumento do risco de problemas } \\
\text { de saúde e bem-estar associado ao isolamento. }\end{array}$ \\
\hline $\begin{array}{l}(2011) \\
\text { Inglaterra }{ }^{(24)}\end{array}$ & $\begin{array}{l}\text { Estudo quantitativo baseado em dados do } \\
\text { Estudo Longitudinal de Envelhecimento Inglês } \\
\text { (ELSA). }\end{array}$ & $\begin{array}{l}\text { Ausência de contato regular com familiares } \\
\text { e amigos e falta de envolvimento em } \\
\text { organizações sociais. }\end{array}$ & $\begin{array}{l}\text { Isolamento social e solidão podem afetar a saúde independentemente. } 0 \text { isolamento } \\
\text { pode afetar o aumento da pressão arterial e os processos inflamatórios associados } \\
\text { ao desenvolvimento de doenças cardiovasculares. }\end{array}$ \\
\hline $\begin{array}{l}\text { (2013) } \\
\text { Inglaterra }{ }^{(25)}\end{array}$ & $\begin{array}{l}\text { Estudo quantitativo baseado em dados do } \\
\text { Estudo Longitudinal de Envelhecimento Inglês } \\
\text { (ELSA). }\end{array}$ & $\begin{array}{l}\text { Ausência de contato regular com familiares } \\
\text { e amigos e falta de envolvimento em } \\
\text { organizações sociais. }\end{array}$ & $\begin{array}{l}0 \text { isolamento foi associado a reduções na fluência verbal, recordação imediata e } \\
\text { atraso na recordação durante um período de quatro anos. Isolamento e solidão } \\
\text { foram significativamente associados com pior função cognitiva. }\end{array}$ \\
\hline $\begin{array}{l}\text { (2015) } \\
\text { Inglaterra' }{ }^{(26)}\end{array}$ & $\begin{array}{l}\text { Estudo quantitativo baseado em dados do } \\
\text { Estudo Longitudinal de Envelhecimento Inglês } \\
\text { (ELSA), com análise inferencial ao longo de } \\
\text { quatro anos. }\end{array}$ & $\begin{array}{l}\text { Ausência de contato regular com familiares } \\
\text { e amigos e falta de envolvimento em } \\
\text { organizaçõos sociais. }\end{array}$ & $\begin{array}{l}0 \text { isolamento e a solidão foram associados, de diferentes formas, a mudanças na } \\
\text { trajetória do bem-estar (avaliativo) cognitivo e afetivo (hedônico) ao longo do tempo. }\end{array}$ \\
\hline $\begin{array}{l}\text { (2018) } \\
\text { Inglaterra }\end{array}$ & \begin{tabular}{|l|} 
Estudo quantitativo baseado em dados do \\
Estudo Longitudinal de Envelhecimento Inglês \\
(ELSA), segunda etapa de coleta de dados \\
(2004-2005), com análise inferencial ao longo \\
de seis anos.
\end{tabular} & $\begin{array}{l}\text { Isolamento social definido de acordo com a } \\
\text { situação conjugal, coabitação, contato com } \\
\text { crianças, parentes, amigos e participação em } \\
\text { organizações sociais. }\end{array}$ & $\begin{array}{l}\text { Baixo nível de alfabetização em saúde e } 0 \text { isolamento foram independentemente } \\
\text { associados ao aumento do risco de mortalidade por todas as causas. Isoladamente, } \\
\text { a baixa alfabetização representou maior chance de morrer em } 22 \% \text { e } 0 \text { isolamento, } \\
28 \% \text {. }\end{array}$ \\
\hline $\begin{array}{l}\text { (2013) } \\
\text { Holanda }^{(28)}\end{array}$ & $\begin{array}{l}\text { Estudo quantitativo baseado em dados de } \\
\text { uma plataforma web do Estudo Longitudinal } \\
\text { na Internet de Ciências Sociais (LISS Panel), } \\
\text { com amostra representativa da população } \\
\text { holandesa. }\end{array}$ & $\begin{array}{l}\text { Definido como uma solidão social decorrente } \\
\text { da ausência de um grupo mais amplo de } \\
\text { contatos e de desengajamento. As medidas } \\
\text { subjetivas envolvem conexão social, } \\
\text { sentimento de isolamento ou desconexão. }\end{array}$ & $\begin{array}{l}\text { Trabalho voluntário, atividades culturais, férias, esportes, leitura, hobbies e compras } \\
\text { são considerados preditores de sucesso para a conexão social das pessoas idosas. } \\
\text { Assistir TV, ouvir rádio e usar o computador não foram associados à conexão social. }\end{array}$ \\
\hline
\end{tabular}

Já a análise de similitude possibilita decifrar as conexóes entre as palavras do corpus textual, favorecendo inferir a construçáo do texto e dos temas relevantes. A figura 2 apresenta uma análise de similitude e descreve as palavras "social", "organização" e "falta", que auxiliaram na identificaçáo do campo representacional das definiçóes atribuídas ao isolamento social. Os resultados indicaram três campos: (i) ausência de contatos e apoio social; (ii) falta fa- miliar; e (iii) falta de envolvimento/organização regular em redes de amigos. A solidão foi fracamente vinculada aos conceitos atribuídos ao isolamento social.

Para a aferição do isolamento social, três artigos utilizaram a Escala de Redes Sociais de Lubben (LSNS), um artigo utilizou a Escala UCLA Loneliness associada a perguntas subjetivas, e todos os demais estudos criaram índices ou instrumen- 


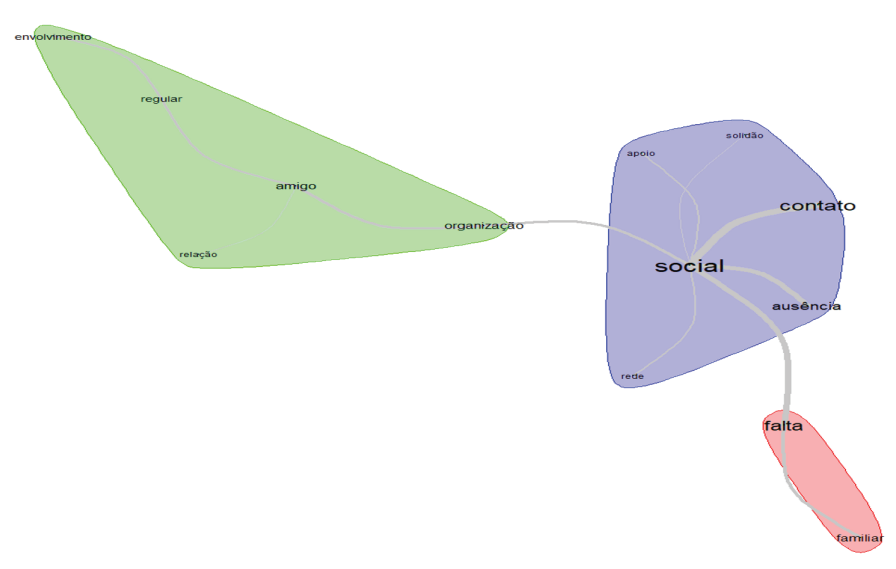

Figura 2. Análise de similitude

tos próprios, representando $78 \%$ dos artigos. Os atributos vinculados à criação desses instrumentos derivaram, principalmente, de: avaliação de status marital; número de filhos; relação familiar; atividade religiosa; participação em grupos (sociais, voluntariado); frequência de contatos; arranjos de moradia e análise geográfica. Entre os principais temas apresentados nos resultados, tem-se avaliação de fatores associados ao: risco de isolamento; possíveis consequências; intervençóes às pessoas idosas isoladas; relação entre isolamento e solidão; e possíveis fatores protetivos.

\section{Discussão}

A partir da análise dos artigos incluídos foi possível identificar um conceito mais geral sobre isolamento social de pessoas idosas, vinculado, de forma objetiva, à escassez de relaçóes humanas e contatos regulares com pessoas, quer sejam familiares, quer sejam amigos ou membros da comunidade. Nesses casos, o indivíduo, no cotidiano de sua vida, interage com um número menor de pessoas do que gostaria, sua rede social é reduzida e conta com insuficiência de apoio social - emocional, informativo e instrumental. Relaciona-se com o histórico de vida e o contexto de organização social. Nessa perspectiva, o isolamento náo se refere àqueles que se desconectaram voluntariamente, mas a possíveis barreiras que dificultam ou impedem a conexáo social.
Algumas pesquisas destacaram a relação do isolamento social com os fatores emocionais. ${ }^{(12,17,21-22)}$ Para eles, o conceito de isolamento é vinculado à falta de interação social objetiva e apresenta-se relacionado com uma percepção subjetiva de isolamento emocional. Esses conceitos aproximam a ausência ou a escassez de interação social do sentimento ou percepção de solidão.

Apesar dessa aproximação conceitual entre isolamento e solidão, estudos ${ }^{(12,14,24-26)}$ sustentam que os construtos são diferentes e não estão necessariamente correlacionados. A solidão não depende da quantidade de interaçóes sociais, configurando-se como uma experiência altamente subjetiva. Já o isolamento está diretamente relacionado à diminuição objetiva das redes sociais. ${ }^{(29)}$ Considerando essas diferenças, os autores indicam a necessidade de distinção dos problemas nas pessoas idosas, para uma intervenção mais específica e direcionada, uma vez que suas consequências à saúde podem ser distintas. ${ }^{(24-26)}$

Entretanto, todas as definiçôes analisadas constatam que, no caso de isolamento social, o número de relacionamentos interpessoais é significativamente diminuído ou mesmo ausente. As formas de medir o isolamento social ainda apresentam um déficit de padronização operacional e este pode ser uma das razóes pelas quais foram encontradas diferentes abordagens conceituais sobre o isolamento e poucos estudos com medidas validadas para além das escalas de solidão. A maioria dos artigos não apresenta a operacionalização do conceito de ma- 
neira a favorecer a mensuração do fenômeno; apenas um artigo ${ }^{(19)}$ discute a importância da questáo cultural a ser usada na construçáo conceitual e a ser usada para programas de intervenção de redução do isolamento social de pessoas idosas.

No Brasil, um artigo publicado(30) a partir do Estudo Longitudinal de Saúde dos Idosos Brasileiros (ELSI-Brasil) evidencia que os aspectos de participação social e percepção de vizinhança são influenciados por características urbanas. Situaçóes urbanas como dificuldades de cruzamento de ruas e acessibilidade ao transporte coletivo foram identificados como barreiras para a participação social. Tais aspectos expressam a heterogeneidade das situaçóes enfrentadas pelas pessoas idosas, o acesso ao direito às cidades e as diferenças entre os países.

Entre as consequências do isolamento social na vida da pessoa idosa destacam-se: maior risco por problemas de saúde, redução do bem-estar e aumento da mortalidade. ${ }^{(23-25,27)}$ Outros achados também podem ser considerados, tais como: prejuízo para saúde cognitiva e comprometimento da saúde mental; (25-26) ampliação dos riscos para depressão e ansiedade; ${ }^{(26)}$ redução da atividade física diária e maior tempo sedentário; ${ }^{(26)}$ pior qualidade do sono; ${ }^{(11)}$ maior chance de desenvolver doenças cardiovasculares ${ }^{(24)}$ e sensação de vida insatisfatória. ${ }^{(21)}$

As consequências podem ser ainda mais impactantes, conforme um estudo realizado no Japão, que detém o maior número de pessoas idosas do mundo contemporâneo. Estima-se que estas irão represen$\operatorname{tar} 40,5 \%$ da população em 2055 . Tem sido identificada a chamada morte solitária como uma das consequências do isolamento social. ${ }^{(1,31)}$

Esta morte solitária acontece quando um indivíduo morre sozinho em casa e isso passa despercebido pelos outros. O corpo do falecido pode ser deixado sem vigilância por dias, meses ou mesmo anos. É considerado um desfecho trágico e representa, simbólica e concretamente, o isolamento social que parte da humanidade está vivenciando. ${ }^{(31)}$

A literatura estudada revela que o isolamento social é potencialmente evitável e que as intervençóes capazes de preveni-la ou atuar na sua superação possuem múltiplas dimensóes. Programas destinados a prevenir o isolamento social são mais eficazes quando utilizam os recursos existentes na comunidade; são feitos sob medida, de acordo com as necessidades do indivíduo e têm como alvo pessoas que podem compartilhar experiências semelhantes. (22) Nesse sentido, os artigos assinalam a necessidade de delimitar bem as singularidades dos indivíduos para se realizar a intervenção.

No estudo realizado em Alabama ${ }^{(13)}$, identificou-se, por exemplo, que a frequência de uso da internet tem o potencial de afetar indivíduos que sofrem de solidão, mas não modificam as percepções de isolamento. No estudo indiano, evidenciou-se que assistir a TV, ouvir rádio e utilizar computador também não foram associados à conexão social. ${ }^{(28)}$ $\mathrm{O}$ contato face a face parece ser o caminho para intervir positivamente no isolamento.

$\mathrm{Na}$ pesquisa realizada nos Países Baixos ${ }^{(19)}$ evidenciou-se que pessoas idosas, com nível de interação baixo há muito tempo, necessitam de maiores motivaçóes e suporte do que aquelas que vivem em isolamento recente, situacional. Diante disso, observa-se que as intervençóes devem considerar desde relacionamentos mal resolvidos até a reconstrução de laços e vínculos, com intervençóes por meio de atividades sociais e grupos de apoio, investindo numa sociabilidade ativa.

Os estudos analisados também apontam fatores protetivos contra a escassez de relaçóes sociais: trabalho voluntário, atividades culturais, férias, esportes, leitura, hobbies, compras, ser cristão ativo e possuir filhos, trabalho e ou aposentadoria foram considerados preditores de sucesso para a conexão social das pessoas idosas. ${ }^{(18,28)}$

Esses países, assim como o Brasil, devem buscar soluçóes alternativas para pessoas idosas se manterem empregadas ou aposentadas, com sistema de previdência estruturado, provisão e alocação de recursos de saúde e comunitários adequados, acesso a oportunidades de interação social e conectividade comunitária e configuração de cidades criativas e interativas que planejam espaços para convívio intergeracional e que possibilitem novas modalidades de interação humana, de forma a afetar positivamente o isolamento vivido pelas pessoas idosas.

Levanta-se como possibilidade analítica, para aprofundamento futuro, a análise entre isolamento social e o construto ageísmo. ${ }^{(32)} \mathrm{O}$ ageísmo possibi- 
lita entender que a narrativa social cravada nas falas populares e nos âmbitos societários mais amplos consolida cotidianamente uma visão de envelhecimento numa perspectiva negativa. Preconceitos e estereótipos disseminados no seio da sociedade interferem na vivência plena da velhice e têm o potencial de interferir na formulação eficaz de políticas que enfrentem os desafios do envelhecimento na contemporaneidade, entre eles, o isolamento social. A própria teoria do desengajamento social como parte do ciclo da vida da pessoa idosa, conforme proposta por Cumming e Henri $^{(33)}$, apresenta um fundo teórico conceitual do envelhecimento que se aproxima do ageísmo e carece de revisão teórica e análise mais profunda.

Um estudo coreano ${ }^{(34)}$ acrescenta uma nova dimensão aos estudos sobre isolamento social quando aponta que o conceito deve também ser pensado na perspectiva da solidão étnica e da diáspora, no qual indivíduos estáo isolados por força de uma experiência de migração, perseguição política ou mesmo abandono. É a experiência de um isolamento por força de uma injustiça ou de subjugação, associado a uma impossibilidade de ser escutado. Essa visão corrobora a possibilidade de relação entre ageísmo, isolamento social e iniquidade social, deixando em aberto possibilidades de novos estudos.

As escalas de identificação do isolamento social carecem de padronização para permitir mensurar e comparar fenômenos entre países. Apesar de existir a escala validada de Lubben, a maior parte dos artigos criou índices próprios para medição que permitem incluir questóes conjunturais e culturais de cada região. A heterogeneidade entre idosos e entre países e suas populaçôes sugere cautela na análise do isolamento social identificado nos estudos.

Devido ao limitado número de bases consultadas e à não inclusão de literatura cinza, não foi possível identificar estudos oriundos de países da América Latina e Caribe, que possuem maior proximidade geográfica, cultural e social com o Brasil.

\section{Conclusão}

Conclui-se que as pesquisas sobre isolamento social de pessoas idosas apresentam lacunas ao descrever os instrumentos adotados, a operacionalização das bases epistemológicas e as perspectivas teóricas do conceito adotado. Os estudos evidenciaram uma heterogeneidade de métodos na tentativa de abordar a multidimensionalidade do isolamento social de pessoas idosas, suas implicaçôes, consequências e fatores protetivos. A presente pesquisa permitiu compreender que o isolamento social é multifacetado e que a pessoa idosa isolada náo apresenta características homogêneas. Esse conjunto de características distintas, com diferentes meios de detectar e intervir no problema, evidencia a necessidade de intensificar e especificar as estratégias para minimizar esse problema societário e ampliar a construção de programas baseados em evidências, com monitoramento e avaliação de sua efetividade para grupos heterogêneos. A solidão e o isolamento foram identificados como dois constructos distintos que não necessariamente estão correlacionados em suas implicaçóes. $\mathrm{O}$ isolamento, independentemente de outros fatores, é capaz de influenciar negativamente nos desfechos de saúde, satisfação e bem-estar de modo singular. As escalas de identificação do isolamento social carecem de padronização para permitir mensurar e comparar fenômenos entre países. Apesar de existir a escala validada de Lubben, a maior parte dos artigos criou índices próprios para medição que permitem incluir questôes conjunturais e culturais de cada região. A diversidade cultural dos países e suas populaçóes sugere cautela na análise do isolamento social identificado nos estudos. A ampliação do papel e da função social da pessoa idosa precisa ser ressignificada, e a sociedade necessita superar a separação dessas pessoas na dinâmica das cidades, incluindo-as organicamente. A alocação de recursos na saúde, o enfrentamento ao ageísmo, o sistema de previdência e soluçóes criativas nas cidades podem colaborar para esse enfrentamento.

\section{Agradecimentos}

À Fundação de Apoio à Pesquisa do Distrito Federal (FAPDF) pelo financiamento do Projeto de pesquisa coordenado por Leides Barroso Azevedo Moura. 


\section{Referências}

1. World Health Organization (WHO). [World aging and health report]. Genebra: WHO; 2015. [cited 2018 Dez 14]. Available from: https:// apps.who.int/iris/bitstream/handle/10665/186468/WHO_FWC_ ALC_15.01_por.pdf?sequence=6. Portuguese.

2. Veras RP, Oliveira M. Aging in Brazil: the building of a healthcare model. Ciênc Saúde Colet. 2018;23(6):1929-36.

3. Miranda GM, Mendes AC, Silva AL. Population aging in Brazil: Current and future social challenges and consequences. Rev Bras Geriatr Gerontol. 2016;19(3):507519.

4. Cudjoe TKM, Roth DL, Szanton SL, Wolff JL, Boyd CM, Thorpe RJ. The Epidemiology of Social Isolation: National Health and Aging Trends Study. J Gerontol B Psychol Sci Soc Sci. 2020 Jan 1;75(1):107-13.

5. Suen I, Gendron TL, Gough M. Social Isolation and the Built Environment: A Call for Research and Advocacy. Public Policy Aging Rep. 2017;27(4):131-5.

6. Kinsella $S$. Older people and social isolation: a review of the evidence. Wirral, England: Wirral Council Business \& Public Health Intelligence Team; 2015. p. 4-15.

7. Dahlberg L, Andersson L, Lennartsson C. Long-term predictors of Ioneliness in old age: results of a 20 -year national study. Aging Ment Health. 2018;22(2):190-6.

8. Holt-Lunstad J, Smith TB, Baker M, Harris T, Stephenson D. Loneliness and social isolation as risk factors for mortality: a meta-analytic review. Perspect Psychol Sci. 2015 10(2):227-37.

9. Galvão CM, Sawada NO, Trevizan MA. Revisão sistemática: recurso que proporciona a incorporação das evidências na prática da enfermagem [Systematic review: a resource that allows for the incorporation of evidence into nursing practice]. Rev Lat Am Enfermagem. 2004;12(3):549-56. Portuguese.

10. Minayo MC. [Qualitative analysis: theory, steps and reliability]. Cien Saude Colet. 2012;17(3):621-6. Portuguese.

11. Yu B, Steptoe A, Niu K, Ku PW, Chen LJ. Prospective associations of social isolation and loneliness with poor sleep quality in older adults. Qual Life Res. 2018;27(3):683-91.

12. Cornwell EY, Waite LJ. Measuring social isolation among older adults using multiple indicators from the NSHAP study. J Gerontol B Psychol Sci Soc Sci. 2009;64 Suppl 1:i38-46.

13. Cotten SR, Anderson WA, McCullough BM. Impact of internet use on Ioneliness and contact with others among older adults: cross-sectional analysis. J Med Internet Res. 2013;15(2):e39.

14. Coyle CE, Dugan E. Social isolation, loneliness and health among older adults. J Aging Health. 2012;24(8):1346-63.

15. Drum JL, Medvene LJ. The social convoys of affordable senior housing residents: Fellow residents and "Time Left". Educ Gerontol. 2017;43(11):540-51.

16. Giuli C, Spazzafumo L, Sirolla C, Abbatecola AM, Lattanzio F, Postacchini D. Social isolation risk factors in older hospitalized individuals. Arch Gerontol Geriatr. 2012;55(3):580-5.

17. Ibrahim R, Abolfathi Momtaz Y, Hamid TA. Social isolation in older Malaysians: prevalence and risk factors. Psychogeriatrics. 2013;13(2):71-9.
18. Kotian DB, Mathews M, Parsekar SS, Nair S, Binu VS, Subba SH. Factors associated with social isolation among the older people in India. J Geriatr Psychiatry Neurol. 2018;31(5):271-8.

19. Machielse A. The heterogeneity of socially isolated older adults: a social isolation typology. J Gerontol Soc Work. 2015;58(4):338-56.

20. Menec VH, Newall NE, Mackenzie CS, Shooshtari S, Nowicki S. Examining individual and geographic factors associated with social isolation and loneliness using Canadian Longitudinal Study on Aging (CLSA) data. PLoS One. 2019;14(2):e0211143.

21. Ramage-Morin PL. Hearing difficulties and feelings of social isolation among Canadians aged 45 or older. Health Rep. 2016;27(11):3-12.

22. Saito T, Kai I, Takizawa A. Effects of a program to prevent social isolation on loneliness, depression, and subjective well-being of older adults: a randomized trial among older migrants in Japan. Arch Gerontol Geriatr. 2012;55(3):539-47.

23. Schrempft S, Jackowska M, Hamer M, Steptoe A. Associations between social isolation, Ioneliness, and objective physical activity in older men and women. BMC Public Health. 2019;19(1):74.

24. Shankar A, McMunn A, Banks J, Steptoe A. Loneliness, social isolation, and behavioral and biological health indicators in older adults. Health Psychol. 2011;30(4):377-85.

25. Shankar A, Hamer M, McMunn A, Steptoe A. Social isolation and Ioneliness: relationships with cognitive function during 4 years of follow-up in the English Longitudinal Study of Ageing. Psychosom Med. 2013;75(2):161-70.

26. Shankar A, Rafnsson SB, Steptoe A. Longitudinal associations between social connections and subjective wellbeing in the English Longitudinal Study of Ageing. Psychol Health. 2015;30(6):686-98.

27. Smith SG, Jackson SE, Kobayashi LC, Steptoe A. Social isolation, health literacy, and mortality risk: Findings from the English Longitudinal Study of Ageing. Health Psychol. 2018;37(2):160-9.

28. Toepoel V. Ageing, leisure, and social connectedness: how could leisure help reduce social isolation of older people? Soc Indic Res. 2013;113(1):355-72.

29. Domènech-Abella J, Mundó J, Haro JM, Rubio-Valera M. Anxiety, depression, Ioneliness and social network in the elderly: Longitudinal associations from The Irish Longitudinal Study on Ageing (TILDA). J Affect Disord. 2019; 246:82-8.

30. Ferreira FR, César CC, Andrade FB, Souza P Junior, Lima-Costa MF, Proietti FA. Aspects of social participation and neighborhood perception: ELSI-Brazil] Rev Saúde Públ. 2018;52 Suppl 2:18s.

31. Tadaka E, Kono A, Ito E, Kanaya Y, Dai Y, Imamatsu Y, et al. Development of a community's self-efficacy scale for preventing social isolation among community-dwelling older people (Mimamori Scale). BMC Public Health. 2016;16(1):1198.

32. Butler RN. Ageism: A foreword. J Soc Issues. 1980;36(2):8-11.

33. Cumming E, Henri WE. Growing old: The process of disengagement. New York: Basic Books; 1961.

34. Wong A, Chau AK, Fang Y, Woo J. Illuminating the psychological experience of elderly loneliness from a societal perspective: a qualitative study of alienation between older people and society. Int J Environ Res Public Health. 2017;14(7):824. 